\title{
Antibacterial activity of Shin'iseihaito (Xin Yi Qing Fei Tang) against Streptococcus pneumoniae
}

\author{
Toru Konishi ${ }^{1}$, Masaaki Minami ${ }^{2}$, Zhixia Jiang ${ }^{3}$, Tetsuya Arai $^{3}$ and Toshiaki Makino $^{1 *}$ \\ 'Department of Pharmacognosy, Graduate School of Pharmaceutical Sciences, Nagoya City University, 3-1 Tanabe-dori, Mizuho-ku, Nagoya 467-8603, Japan. \\ 2Department of Bacteriology, Graduate School of Medical Sciences, Nagoya City University, 1 Kawasumi, Mizuho-cho, Mizuho-ku, Nagoya 467-8601, Japan. \\ ${ }^{3}$ Research and Development Center, Kobayashi Pharmaceutiucal Co. Ltd., 4-10 Doshomachi 4-chome, Chuo-ku, Osaka, Japan.
}

\begin{abstract}
Objective: Shin'iseihaito (Xin yi qing fei tang in Chinese, SSHT), a formula in traditional Japanese Kampo medicine and Chinese medicine comprising nine crude drugs, Gypsum, Ophiopogon Tuber, Scutellaria Root (SR, root of Scutellaria baicalensis), Gardenia Fruit, Anemarrhena Rhizome, Lilium Bulb, Magnolia Flower, Loquat Leaf, and Cimicifuga Rhizome, is commonly used to treat sinusitis associated with purulent nasal discharge and reddish nasal mucosa. We evaluated anti-bacterial activity of SSHT extract on Streptococcus pneumoniae, the major cause of bacterial sinusitis. Materials and Methods: Sterile paper disks impregnated with SSHT extract or each extract of its component were placed on sheep blood agar plates inoculated with Streptococcus pneumoniae (ATCC 49619). The diameter of inhibitory zone was measured after 20-24 h incubation. Results: SSHT extract and each water extract of Ophiopogon Tuber, SR, Gardenia Fruit, Anemarrhena Rhizome, Lilium Bulb, Magnolia Flower, and Cimicifuga Rhizome showed significant antibacterial activity against Streptococcus pneumoniae, and SR extract exhibited the largest inhibitory zone. SR extract was partitioned into $\mathrm{AcOEt}, \mathrm{BuOH}$, and water layer, and water layer was further separated into $80 \% \mathrm{EtOH}$ soluble and insoluble fraction. Among them, only $80 \%$ $\mathrm{EtOH}$ soluble fraction was exhibited antibacterial activity. In this fraction, we found baicalin as the major compounds, and baicalin exhibited antibacterial activity against Streptococcus pneumoniae in concentration-
\end{abstract}

dependent manner. Conclusion: SSHT has antibacterial activity against Streptococcus pneumoniae, and SR and its major constituent baicalin contribute to the antibacterial activity of SSHT against Streptococcus pneumoniae.

Key words: Antibacterial activity, Baicalin, Shin'iseihaito, Sinusitis, Streptococcus pneumoniae, Scutellaria baicalensis.

Key Messages: Shin'iseihaito, a formula of traditional Kampo and Chinese medicine, might be effective on sinusitis caused by Streptococcus pneumoniae. Roots of Scutellaria baicalensis, one of the component of shin'iseihaito, and its major constituent baicalin were the active ingredient of in Shin'iseihaito.

Address for correspondence:

Prof. Toshiaki Makino, Department of Pharmacognosy, Graduate School of Pharmaceutical Sciences,Nagoya City University, 3-1 Tanabe-dori, Mizuhoku, Nagoya 467-8603, Japan.

Phone no: +8152836 3416

Email: makino@phar.nagoya-cu.ac.jp

DOI: 10.5530/pj.2016.1.4

\section{INTRODUCTION}

Sinusitis is one of the most common diseases, and affects quality-of-life ${ }^{1}$ as a result of symptoms such as nasal discharge, nasal congestion, hyposmia, facial pain or pressure, headache, and malaise. The major cause of sinusitis is the infection of Streptococcus pneumoniae. ${ }^{2}$ Streptococcus pneumoniae belongs to gram-positive bacteria, and is one of indigenous bacteria present in oral and nasal cavity even in healthy people. Streptococcus pneumoniae is also the most common cause for pneumoniae, meningitis, and otitis media. ${ }^{2}$ Recently, Streptococcus pneumoniae that has antimicrobial resistance has been increasing, and the treatment other than antibiotic therapy is demanded. ${ }^{3}$

Shin'iseihaito (xin yi qing fei tang in Chinese, SSHT) is a formula in traditional Japanese Kampo medicine and Chiniese medicine comprising nine crude drugs, Gypsum (G), Ophiopogon Tuber (OT), Scutellaria Root (SR), Gardenia Fruit (GF), Anemarrhena Rhizome (AR), Lilium Bulb (LB), Magnolia Flower (MF), Loquat Leaf (LL) and Cimicifuga Rhizome (CR). ${ }^{4}$ In Kampo medicine, SSHT is commonly used to treat sinusitis associated with lung-heat syndrome, which is characterized by the symptoms of purulent nasal discharge, reddish nasal mucosa, and so on. ${ }^{4}$ In our previous study, we investigated the preventive effect of SSHT extract in ovalbumin-induced allergic rhinitis model. ${ }^{5}$ However, there are few experimental evidences about the effects of SSHT for the treatment of bacterial sinusitis. Several Kampo formulas and crude drugs have already been known to have antibacterial activity against various bacterial species. ${ }^{6-10}$ However, the antibacterial activity of SSHT against Streptococcus pneumoniae has been unclear.
In this study, we evaluated the antibacterial activities of SSHT hot water extract and its constituents against Streptococcus pneumoniae in vitro. We found that SR contributed to the anti-bacterial effect of SSHT, and we isolated baicalin from SR as its active ingredient.

\section{MATERIALS AND METHODS}

\section{Preparation of herbal formulas}

All crude drugs used in this study have the grade of Japanese Pharmacopoeia $16^{\text {th }}$ Edition. ${ }^{11}$ Gypsum (lot No. 0I02M, natural hydrous calcium sulfate, G), Ophiopogon Tuber (lot No. 4C03M, the tuber of Ophiopogon japonicus, OT), Scutellaria Root (lot No. 0J08M, the root of Scutellaria baicalensis, SR), Gardenia Fruit (lot No. 9I11M, the fruit of Gardenia jasminoides, GJ), Anemarrhena Rhizome (lot No. 0E22, the rhizome of Anemarrhena asphodeloides, AR), and Cimicifuga Rhizome (lot No. 0F28M, the rhizome of Cimicifuga heracleifolia, CR) were purchased from Daiko Shoyaku (Nagoya, Japan). Lilium Bulb (lot No. 19026391, the bulb of Lilium lancifolium, LB), Magnolia Flower (lot No. 19034071, the flower of Magnolia salicifolia, MF), and Loquat Leaf (lot No. 19030171, the leaf of Eriobotrya japonica, LL) were purchased from Tsumura Co. Ltd. (Tokyo, Japan). Each crude drug ( $5 \mathrm{~g}$ ) or the mixture of crude drugs constituting SSHT (total $27 \mathrm{~g}$, Table 1) were boiled in 20-times weight of distilled water for $30 \mathrm{~min}$ and filtrated, lyophilized, and the dried powdered extracts of each crude drug or SSHT were weighed. The yields of the extracts to the original crude drugs were shown in Table 1 . The powdered 


\section{Table 1 : Compositions of shin'iseihaito (SSHT)}

\begin{tabular}{|c|c|c|}
\hline Name of crude druga & Weight $^{b}(g)$ & Ratio of yield' (\%) \\
\hline Gypsum (G) & 5.0 & 1.6 \\
\hline $\begin{array}{c}\text { Ophiopogon Tuber } \\
\text { (OT) }\end{array}$ & 5.0 & 36 \\
\hline Scutellaria Root (SR) & 3.0 & 42 \\
\hline $\begin{array}{l}\text { Anemarrhena } \\
\text { Rhizome (AR) }\end{array}$ & 3.0 & 32 \\
\hline Gardenia Fruit (GF) & 3.0 & 37 \\
\hline Lilium Bulb (LB) & 3.0 & 20 \\
\hline Magnolia Flower (MF) & 3.0 & 14 \\
\hline Loquat Leaf (LL) & 2.0 & 19 \\
\hline $\begin{array}{l}\text { Cimicifuga Rhizome } \\
\text { (CR) }\end{array}$ & 1.0 & 18 \\
\hline Shin'iseihaito (SSHT) & Mixture of above ( $27 \mathrm{~g}$ ) & 26 \\
\hline
\end{tabular}

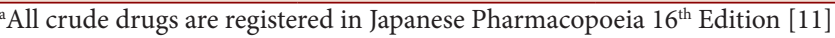

${ }^{b}$ Weight of each herbal medicine in a daily dosage of SSHT.

'The decoctions were prepared according to experimental design, and ratio of yield means $\%$ of dried weight of the decoction to the original crude drug.

extracts were stored in desiccated condition until use. Baicalin was purchased from Wako Pure Chemical Industries (Osaka, Japan).

SSHT extract $(50 \mathrm{mg})$ was suspended with $\mathrm{MeOH}(1 \mathrm{~mL})$ and sonicated for $30 \mathrm{~min}$. The supernatant $(30 \mu \mathrm{L})$ was injected to HPLC with the following conditions: system, Shimadzu LC-10A TSK-GEL ODS- $80_{\text {TS }}(4.6 \times 250 \mathrm{~mm}$, Tosoh, Tokyo); mobile phase, $0.05 \mathrm{M}$ $\mathrm{AcOH}-\mathrm{AcONH}_{4}$ buffer ( $\left.\mathrm{pH} 3.6\right) / \mathrm{CH}_{3} \mathrm{CN}$ 90:10 (0 min)-45:55 (40 min), linear gradient; flow rate, $1.0 \mathrm{~mL} / \mathrm{min}$; column temperature, $40^{\circ} \mathrm{C}$; and detection, $200-400 \mathrm{~nm}$ by a photodiode array detector. Some peaks were identified by the retention times and UV spectra of the standard compounds. The fingerprint chromatogram of SSHT extract is shown in Figure 1.

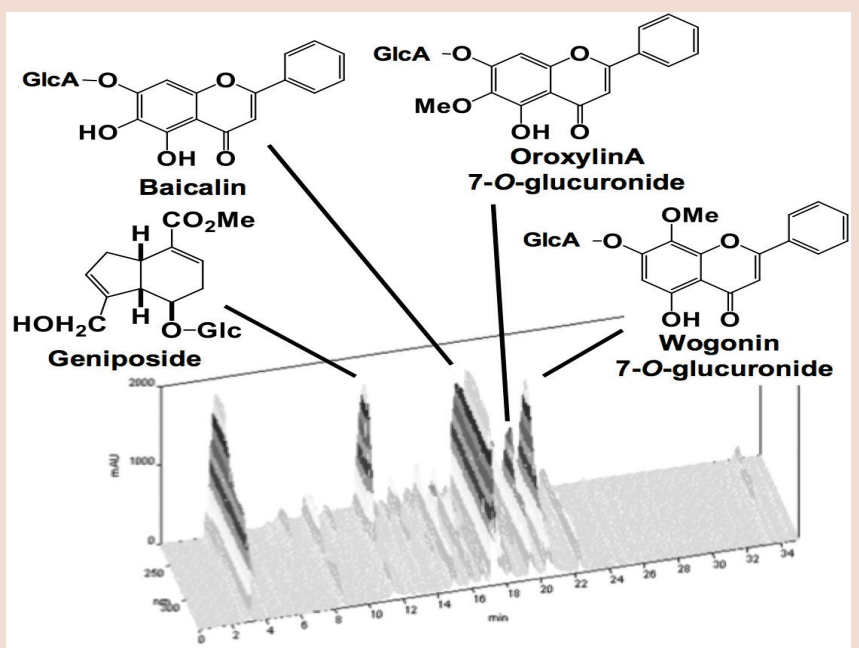

Figure 1: HPLC fingerprint of SSHT extract. SSHT extract $(50 \mathrm{mg})$ was suspended with $\mathrm{MeOH}(1 \mathrm{~mL}$ ) and sonicated for $30 \mathrm{~min}$.

The supernatant $(30 \mu \mathrm{L})$ was injected to HPLC with the following conditions: column, TSK-GEL ODS80 $0_{\mathrm{TS}}(4.6 \times 250 \mathrm{~mm})$; mobile phase, $0.05 \mathrm{M} \mathrm{AcOH}-$ AcONH ${ }_{4}$ buffer ( $\left.\mathrm{pH} 3.6\right) / \mathrm{CH}_{3} \mathrm{CN} 90: 10$ (0 min)-45:55 (40 min), linear gradient; flow rate, $1.0 \mathrm{~mL} / \mathrm{min}$; column temperature, $40^{\circ} \mathrm{C}$; detection, $200-400 \mathrm{~nm}$ by a photodiode array detector. Some peaks were identified by the retention times and UV spectra of the standard compounds.

\section{Bacterial strains and disk diffusion method}

Streptococcus pneumoniae (ATCC49619) was purchased from American Type Culture Collection (Rockville, MD, USA). ATCC49619 was isolated from sputum of 75-year-old male, Phoenix, AZ, and minimal inhibitory concentration of penicillin G for ATCC49619 was $0.25 \mu \mathrm{g} /$ $\mathrm{mL}$. Antibacterial activity was evaluated by the disk diffusion method. ${ }^{12}$ Kirby-Bauer Disk 'EIKEN' containing penicillin G (10 U=6 $\mu \mathrm{g} /$ disk $)$ (Eiken Kagaku, Tokyo, Japan) was used as the positive control. Sterile paper disks were impregnated with SSHT extract, each crude drug extract, each fraction of SR extract, or baicalin, and dried up at room temperature. The colonies of Streptococcus pneumoniae which cultured overnight on Trypticase ${ }^{\mathrm{TM}}$ soy agar with $5 \%$ sheep blood (Nippon Becton Dickinson Co., Ltd., Tokyo, Japan) at $37^{\circ} \mathrm{C}$ under $5 \% \mathrm{CO}_{2}$ atmosphere, were collected into Columbia broth (3.5 g/L) (Nippon Becton Dickinson) at the density of Streptococcus pneumoniae, $2.0 \times 10^{9}$ Colony-forming units $(\mathrm{CFUs}) / \mathrm{mL}$, and inoculated on another Trypticase ${ }^{\mathrm{TM}}$ soy agar with $5 \%$ sheep blood. The paper disks were placed on the plates, and were incubated at $37^{\circ} \mathrm{C}$ under $5 \% \mathrm{CO}_{2}$ atmosphere for $20-24 \mathrm{~h}$. Then, the diameter of inhibitory zone was measured.

\section{Fractionation of scutellaria root extract}

Hot water extract of SR (33 mg) was suspended in water, and extracted with ethyl acetate (AcOEt), then, water layer was extracted with watersaturated n-buthanol $(\mathrm{BuOH})$. Four-times volume of EtOH was dropped into the water layer, and was centrifuged to yield $80 \% \mathrm{EtOH}$ soluble and insoluble fractions. All fractions were lyophilized to yield $1.6 \mathrm{mg}, 2.9$ $\mathrm{mg}, 27.4 \mathrm{mg}$, and $5.2 \mathrm{mg}$ of AcOEt, $\mathrm{BuOH}, 80 \% \mathrm{EtOH}$ soluble, and $80 \%$ $\mathrm{EtOH}$ insoluble fractions, respectively.

HPLC analysis for SR extract and its each fraction was conducted according to the protocol described in our previous article. ${ }^{13}$ SR extract and its fractions were dissolved in distilled water at the concentration of $50 \mathrm{mg} / \mathrm{mL}$ as original SR, and the supernatant $(10 \mu \mathrm{L})$ was injected to HPLC with the following conditions: system, Elite LaChrom (Hitachi, Tokyo, Japan); column, COSMOSIL 5C ${ }_{18}$ AR-II (4.6×150 mm, Nakarai, Kyoto); mobile phase, A: diluted phosphoric acid (1 in 146), B: acetonitrile, $A: B=72: 28$; flow rate, $0.7 \mathrm{~mL} / \mathrm{min}$; column temperature, $50^{\circ} \mathrm{C}$; and detection, UV $277 \mathrm{~nm}$ by a photodiode array detector. Retention time of baicalin was $5.7 \mathrm{~min}$. Calibration line of baicalin standard was achieved by linear regression analysis, using the least squares method from the peak areas obtained $\left(r^{2}=0.995 ; 7.25-100 \mu \mathrm{g} / \mathrm{mL}\right.$ for baicalin $)$.

\section{Statistical Analysis}

All statistical analyses were performed using the PASW Statistics version 18 (SPSS, IBM, Armonk, NY, USA). The statistical analysis was conducted using a one-way analysis of variance (ANOVA) followed by Bonferroni/Dunnett's multiple $t$-test for the differences among multiple groups. $P$-values less than 0.05 were considered statistically significant.

\section{RESULTS AND DISCUSSION}

Antibacterial activity of SSHT extract against Streptococcus pneumoniae was evaluated by using disk diffusion method. As shown in Figure 2a, SSHT extract (3.8-7.5 mg/disk) exhibited antibacterial activity against Streptococcus pneumoniae in a dose-dependent manner. SSHT extract (7.5 mg/disk) was showed equal antibacterial activity compared with penicillin $\mathrm{G}(6 \mu \mathrm{g} /$ disk$)$.

We screened the antibacterial activity of the hot water extracts of nine crude drugs composing SSHT (Figure $2 \mathrm{~b}$ ). Water extracts of SR at 7.5 $\mathrm{mg}$ /disk exhibited the strongest antibacterial activity against Streptococcus pneumoniae, and in other components prescribed in SSHT, the extracts of CR, OT, GF, AR, LB, and MF showed significant antibacterial activity against Streptococcus pneumoniae in order of higher activity. In traditional pharmacological theory described in the textbook, ${ }^{14}$ SR clears heat and dries dampness, resolves toxicity on the upper burner, and calms the fetus, and CR discharges exterior condition, clears heat, and resolves toxicity. In other crude drugs, OT, GF, AR, and G among 


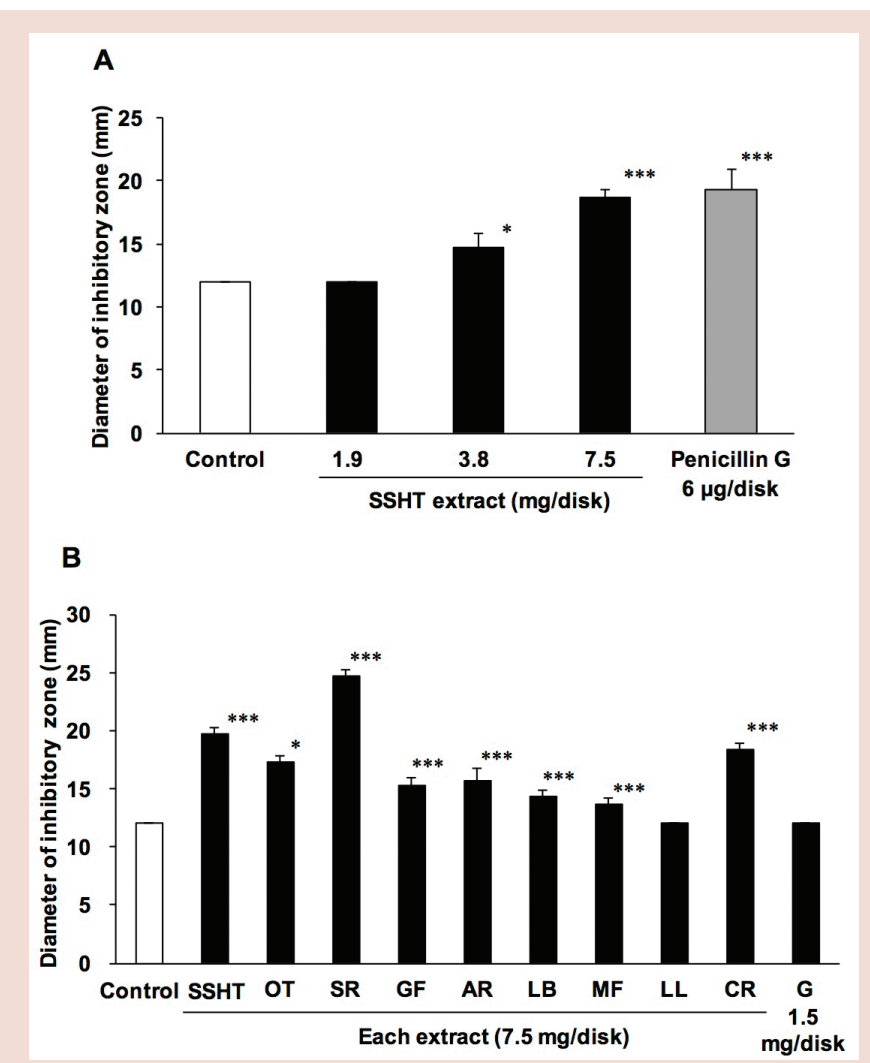

Figure 2: Antibacterial effects of the extracts of SSHT (A) or 9 crude drugs consisting of SSHT (B) against Streptococcus pneumoniae. Paper disks containing the extracts of SSHT, Ophiopogon Tuber (OT), Scutellaria Root (SR), Gardenia Fruit (GF), Anemarrhena Rhizome (AR), Lilium Bulb (LB), Magnolia Flower (MF), Loquat Leaf (LL), Cimicifuga Rhizome (CR), Gypsum (G), or penicillin $\mathrm{G}$ were put on placed on the plates inoculated with Streptococcus pneumoniae, and the diameter of inhibitory zone after 20-24 $\mathrm{h}$ incubation was measured. Values are expressed as the mean \pm S.D. $(n=3)$. ${ }^{*} P<0.05$, ${ }^{* * *} P<$ 0.001 vs control group evaluated by Bonferroni/Dunnet's multiple $t$-test.

the components of SSHT also clear heat. Gypsum $(\mathrm{G})$ is a mineral crude drug, and it is not active in a single use but supports the other combined crude drugs such as AR. ${ }^{15}$ Therefore, the term heat in traditional medicinal theory would be related to bacterial infection, and the antibacterial activity of SSHT exhibited in the present study would be supplied by crude drugs prescribed in SSHT that clear heat in traditional theory.

Since SR extract exhibited the largest inhibitory zone $(25 \mathrm{~mm})$ among water extracts of nine crude drugs $(7.5 \mathrm{mg} /$ disk), we focused SR that is considered to be mainly contributed the antibacterial activity of SSHT extract against Streptococcus pneumoniae. The hot water extract of SR (1.9-7.5 mg/disk) exhibited antibacterial activity against Streptococcus pneumoniae in a dose-dependent manner, and SR extract (3.8 mg/disk) showed equally antibacterial activity compared with penicillin $\mathrm{G}(6 \mu \mathrm{g} /$ disk) (Figure 3a). It is reported that the hot water extract of SR had the antibacterial activity against Propionibacterium acnes ${ }^{6}$ and Helicobacter pylori, ${ }^{7}$ and $60 \%$ ethanol extract of SR had antibacterial activity against Staphylococcus aureus, Listeria monocytogenes, and Salmonella enterica serovars Kentucky, Senftenberg, Enteritidis and Typhimurium. ${ }^{10}$ P. acnes and Streptococcus pneumoniae belong to gram-positive bacteria, but $H$. pylori and Salmonella enterica are gram negative. It is suggested that SR water extract would have broad spectrum anti-bacterial avtivity.

We partitioned the hot water extract of SR into AcOEt, $\mathrm{BuOH}, 80 \%$ $\mathrm{EtOH}$ soluble and insoluble fractions, and evaluated the antibacterial activities against Streptococcus pneumoniae. The dose of each fraction

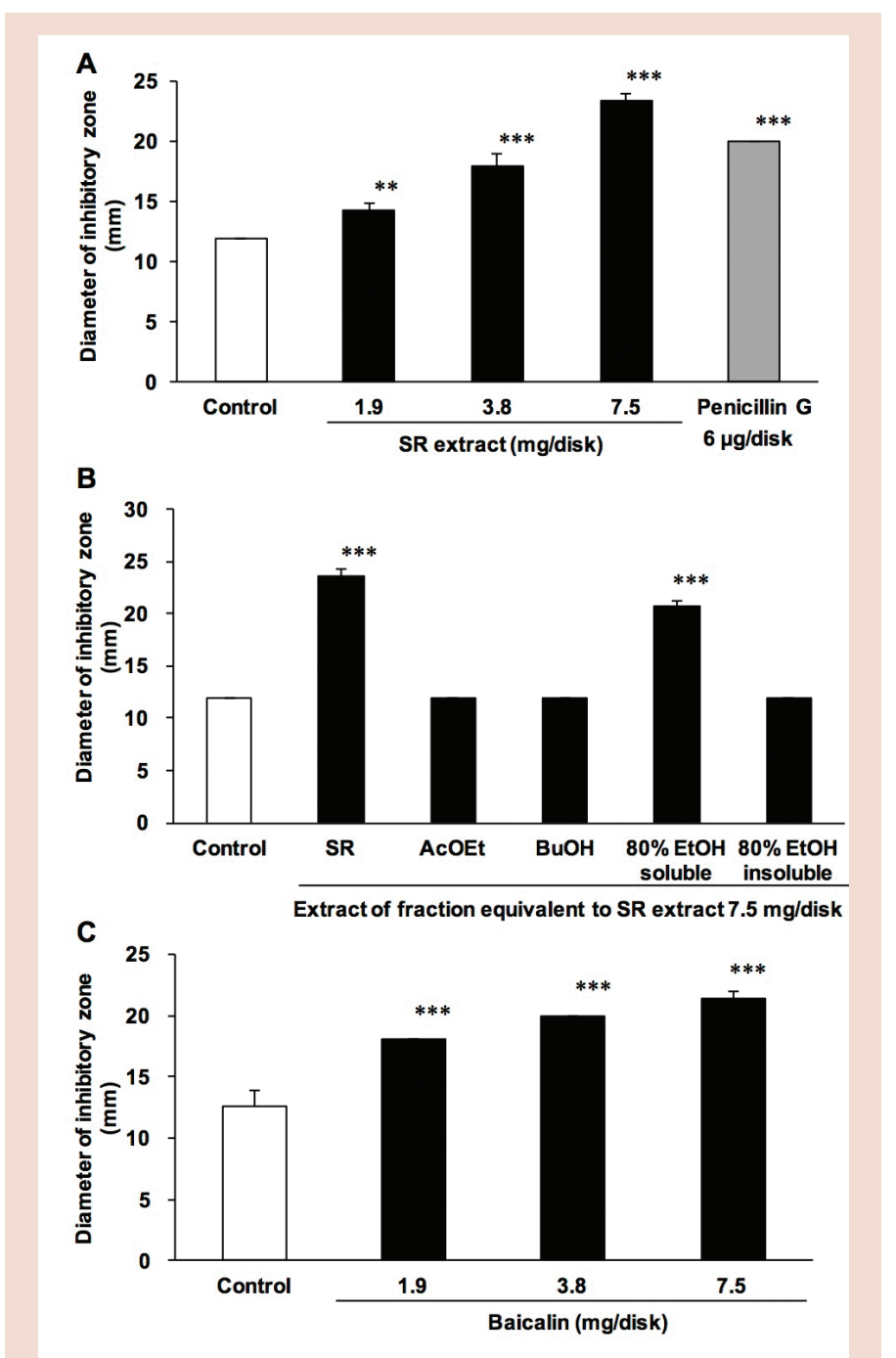

Figure 3: Antibacterial effect of (A) the extract of $S R$, (B) fractions of the extract of $S R$, and (C) baicalin against Streptococcus pneumoniae. Paper disks containing SR extract, its fractions, or baicalin were put on placed on the plates inoculated with Streptococcus pneumoniae, and the diameter of inhibitory zone after 20-24 $\mathrm{h}$ incubation was measured. Each fraction was equivalent to SR extract ( $7.5 \mathrm{mg} /$ disk). Values are expressed as the mean \pm S.D. $(n=3) .{ }^{*} P<0.01$, ${ }^{* * *} P<0.001$ vs control group evaluated by Bonferroni/Dunnet's multiple $t$ test.

corresponded to SR extract (7.5 mg/disk). Among these fractions, only $80 \% \mathrm{EtOH}$ soluble fraction exhibited the antibacterial activity against Streptococcus pneumoniae (Figure $3 \mathrm{~b}$ ).

We measured the amount of baicalin in these fractions using HPLC because baicalin is the marker compounds of $\mathrm{SR}^{11}$ and has the antibacterial activities against Sarcina lutea, ${ }^{9}$ and against Staphylococcus aureus, Listeria monocytogenes, and Salmonella enterica serovars Kentucky, Senftenberg, Enteritidis and Typhimurium. ${ }^{10}$ We confirmed that $80 \% \mathrm{EtOH}$ soluble fraction contained the most of baicalin but other fractions did not (data not shown). This result was consistent with the results of the antibacterial activity of these fractions. Then, we quantitated the amount of baicalin in hot water extract of SR by HPLC, and baicalin content in SR extract was $33(\mathrm{w} / \mathrm{w}) \%$. This means $7.5 \mathrm{mg}$ of SR extract includes 2.5 $\mathrm{mg}$ of baicalin. Then, we examined the antibacterial activity of baicalin against Streptococcus pneumoniae. Baicalin (1.9-7.5 mg/disk) exhibited significant antibacterial activity against Streptococcus pneumoniae in a 
dose-dependent manner (Figure 3c). The inhibitory zone of baicalin ( $2.5 \mathrm{mg} /$ disk) was approximately $19 \mathrm{~mm}$, and activity inhibitory zone was $7 \mathrm{~mm}$ (subtract the inhibitory zone of control $(12 \mathrm{~mm})$ ). The inhibitory zone of SR extract ( $7.5 \mathrm{mg} /$ disk) was approximately $23 \mathrm{~mm}$, and activity inhibitory zone was $11 \mathrm{~mm}$. Though SR contains other compounds than baicalin, baicalin would mainly contribute to the antibacterial activity of SR extract.

\section{CONCLUSION}

The hot water extract of SSHT, a formula in traditional Japanese Kampo medicine, showed the antibacterial activity against Streptococcus pneumoniae, and among the components of SSHT, the extract of SR exhibited the strongest the activity. Baicalin is mainly contributes to the antibacterial activity of SR extract against Streptococcus pneumoniae. Although SSHT contains other crude drugs than SR that have antibacterial activity and these crude drugs might exhibit synergistic antibacterial activity against Streptococcus pneumoniae, this effect of crude drugs might be related to one of the action mechanisms of SSHT to treat bacterial sinusitis.

\section{CONFLICT OF INTEREST}

This study was funded by Kobayashi Pharmaceutical Co.

\section{ABBREVIATION USED}

ANOVA: A one-way analysis of variance; AR: Anemarrhena Rhizome; CR: Cimicifuga Rhizome; GF: Gardenia Fruit; G: Gypsum; LB: Lilium Bulb; LL: Loquat Leaf; MF: Magnolia Flower; OT: Ophiopogon Tuber; SR: Scutellaria Root; SSHT: Shin'iseihaito (xin yi qingfei tang in Chinese).

\section{REFERENCES}

1. Metson RB, Gliklich RE. Clinical outcomes in patients with chronic sinusitis. The Laryngoscopen 2000; 110(S94): 24-8.

2. Brook I. Microbiology of sinusitis. Proc Am Thorac Soc. 2011; 8(1): 90-100.

3. Feldman C, Anderson R. New insights into pneumococcal disease. Respirol. 2009; 14(2): 167-79.

4. The Japan Society for Oriental Medicine. Introduction to Kampo. Tokyo, Japan Elsevier Japan KK; 2005.

5. Minami M, Konishi T, Jiang Z, Arai T, Makino T. Effect of shin'iseihaito (xinyiq ingfeitang) on murine allergic reaction induced by nasal sensitization. J. Trad. Comp. Med. doi:10.1016/j.jtcme.2015.06.001

6. Higaki S, Nakamura M, Morohashi M, Hasegawa Y, Yamagishi T. Activity of eleven kampo formulations and eight kampo crude drugs against Propionibacterium acnes isolated from acne patients: retrospective evaluation in 1990 and 1995. J Dermatol. 1996; 23(12): 871-5

7. Ma F, Chen Y, Li J, Qing HP, Wang JD, Zhang YL, et al. Screening test for antiHelicobacter pylori activity of traditional Chinese herbal medicines. World $J$ Gastroenterol. 2010; 16(44): 5629-34.

8. Gupta VK, Fatima A, Faridi U, Negi AS, Shanker K, Kumar JK, et al. Antimicrobia potential of Glycyrrhiza glabra roots. J Ethnopharmacol. 2008; 116(2): 377-80.

9. Kubo M, Kimura Y, Odani T, Tani T, Namba K. Studies on Scutellariae Radix. Part II: The antibacterial substance. Planta Med. 1981; 43(10): 194-201.

10. Lu $Y$, Joerger R, Wu C. Study of the chemical composition and antimicrobial activities of ethanolic extracts from roots of Scutellaria baicalensis Georgi. J Agric Food Chem. 2011; 59(20): 10934-42.

11. The Society of Japanese Pharmacopoeia. Japanese Pharmacopoeia $16^{\text {th }}$ EdTokyo, Japan: Yakuji-Nippo; 2011.

12. Clinical and Laboratory Standards Institute. Performance standards for antimicrobial disk susceptibility tests; approved standard - eleventh edition CLSI document M02-A11 Wayne, Pennsylvania, USA; 2012.

13. Makino T, Hishida A, Goda Y, Mizuami H. Comparison of the major flavonoid content of $S$. baicalensis, S. lateriflora, and their commercial products. J Nat Med. 2008; 62(3): 294-9.

14. Bensky D, Clavey S, Stoger E. Chinese herbal medicine Materia Medica $3^{\text {rd }}$ Ed. Eastland Press, Seatlle, WA, USA; 1996.

15. Makino $T$, Shiraki $Y$, Mizukami $H$. Interaction of gypsum and the rhizome of Anemarrhena asphodeloides plays an important role in anti-allergic effects of byakkokakeishito in mice. J Nat Med. 2014; 68(3): 505-12.
PICTORIAL ABSTRACT

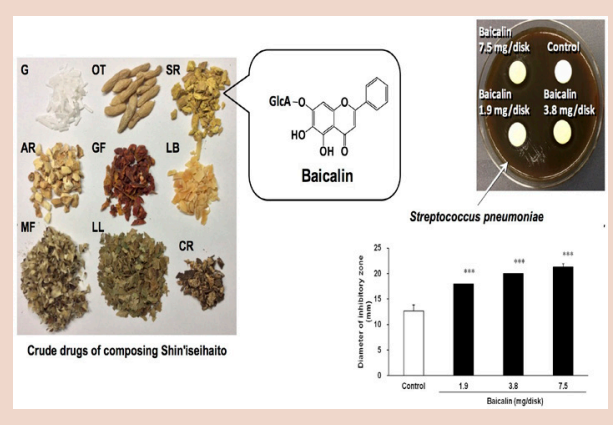

\section{SUMMARY}

- Shin'iseihaito (xin yi qing fei tang in Chinese, SSHT), a formula in traditional Japanese Kampo medicine and Chinese medicine comprising nine crude drugs.

- SSHT extract and each water extract of Ophiopogon Tuber, Scutellaria Root, Gardenia Fruit, Anemarrhena Rhizome, Lilium Bulb, Magnolia Flower, and Cimicifuga Rhizome showed significant antibacterial activity against Streptococcus pneumoniae, and Scutellaria Root extract exhibited the largest inhibitory zone.

- Baicalin as the major compounds in Scutellaria Root exhibited antibacterial activity against Streptococcus pneumoniae in concentration-dependent manner.

\section{ABOUT AUTHOR}

Toru Konishi: Is a Ph. D. student at Department of Pharmacognosy, Graduate School of Pharmaceutical Sciences, Nagoya City University. He graduated in Bachelorof Faculty of Pharmaceutical Sciences, Hokkaido University, and got a license of Pharmacist in Japan. His doctoral research focused on the evaluation of anti-rhinitis activity of Japanese traditional Kampo medicine. 